\title{
Relative Efficacy and Economics of Bio-pesticides against Spodoptera litura (Fab.) on Cabbage
}

\section{G.C. Jat ${ }^{1 *}$, R. Swaminathan ${ }^{1}$, P.C. Yadav ${ }^{1}$, Swati $^{2}$, H.L. Deshwal ${ }^{3}$, Suman Choudhary ${ }^{4}$ and Suresh Kumar Yadav ${ }^{1}$}

\author{
${ }^{1}$ Department of Entomology, ${ }^{2}$ Department of Molecular Biology and Biotechnology, Rajasthan \\ College of Agriculture, (MPUAT) Udaipur, (Rajasthan) 313001, India \\ ${ }^{3}$ Department of Entomology, College of Agriculture and Agriculture Research Station, (SKRAU) \\ Bikaner, (Rajasthan), India \\ ${ }^{4}$ Department of Entomology, SKN College of Agriculture, (SKNAU) Jobner, \\ Jaipur (Rajasthan) 303329, India \\ *Corresponding author
}

\section{A B S T R A C T}

The field experiment on "Relative efficacy and economics of bio-pesticides" was conducted during 2012-13 and 2013-14 at Horticulture farm and Department of Entomology Rajasthan College of Agriculture, (MPUAT) Udaipur (Rajasthan) during rabi 2012-13 and 2013-14. The relative efficacy of three biopesticides viz.,Spinosad, Bt.k. and

\section{Keywords}

Spodoptera litura (Fab.), SINPV, Spinosad, Bt.k. and Cabbage variety Golden acre.

\section{Article Info}

Accepted:

23 May 2017

Available Online:

10 June 2017 $S I N P V$ in alone and in different combinations in nine different schedule (Spinosad-45SC at 200g/ha, Bt.k. (Dipel 8L) at 1lit, SINPV-250LE at 250ml/ha, Spinosad-45SC at 200g/hSINPV-250LE at $250 \mathrm{ml} / \mathrm{ha} S I N P V-250 \mathrm{LE}$ at $250 \mathrm{ml} / \mathrm{ha}$, Spinosad-45SC at $200 \mathrm{~g} / \mathrm{ha}-$ Bt.k.(Dipel 8L) at 1lit -Bt.k-(Dipel 8L) at 1lit., SINPV-250LE at 250ml/ha- Spinosad-45SC at 200g/ha- Spinosad 45SC at 200g/ha, SINPV-250LE at 250ml/ha Bt.k.- (Dipel 8L) at 1litBt.k.. (Dipel 8L) at 1lit, Bt.k.- (Dipel 8L) at 1lit Spinosad-45SC at 200g/ha-Spinosad-45SC at $200 \mathrm{~g} / \mathrm{ha}, B t . k .-$ (Dipel 8L) at 1 lit-SlNPV-250LE at $250 \mathrm{ml} / \mathrm{ha}-S I N P V-250 \mathrm{LE}$ at $250 \mathrm{ml} / \mathrm{ha}$ ) was evaluated against $S$. litura revealed that treatment schedule comprising three spray of spinosad $45 \mathrm{SC}$ at $200 \mathrm{~g} / \mathrm{ha}$ at 15 days interval was found most effective in reducing larval population up to 80.33 and 80.88 per cent during 2012-13 and 2013-14, respectively. It also recorded that higher head yield of 287.35 and $291.15 \mathrm{q} \mathrm{ha}^{-1}$ during rabi 2012-13 and 2013-14, respectively. The treatment schedule comprising three spray of Bt.k. (Dipel 8L) at 1 lit/ha at 15 days interval was found least effective which caused the minimum reduction of larval population with the mean of 56.09 and 55.24 per cent during rabi 201213 and 2013-14, respectively. The highest benefit: cost ratio of 1.419 and 1.407 was recorded in treatment schedule $\mathrm{T}_{1}$ copmprising three spray of spinosad $45 \mathrm{SC}$ at $200 \mathrm{~g} / \mathrm{ha}$ at 15 days interval during rabi 2012-13 and 2013-14, respectively.

\section{Introduction}

Cruciferous vegetables have an important place among rabi crops grown in India. Cabbage, Brassica oleracea var. capitata (Linn.), is a popular vegetable that is grown in all the states of India and has appreciable nutritional and economic value. Cabbage is used as salad, boiled vegetable, in curries and pickles; it is rich in minerals and vitamin A, 
$\mathrm{B}_{1}, \mathrm{~B}_{2}$ and $\mathrm{C}$. The more important insect pests that infest cabbage crop are the tobacco caterpillar (Spodoptera litura Fab.), diamond back moth (Plutella xylostella L.), cabbage semilooper (Trichoplusia ni Hubner), painted bug (Bagrada hilaris Burmeister and Bagrada cruciferarum Kirk.), cabbage butterfly (Pieris brassicae L.), flea beetle (Phyllotreta cruciferae Goeze), aphids (Lipaphis erysimi Kalt. and Brevicoryne brassicae L.), Cabbage leaf webber (Crocidolomia bionotalis Zell) and the mustard saw fly (Athalia lugens proxima Klug.) (Ayyar, 1963; Lall, 1964; Choudhari et al., 2001, and Rao and Lal, 2005). Among these, Spodoptera litura (F.) (Lepidoptera: Noctuidae), is a major pest of cabbage. The pest causes damage to an extent of 80-100 per cent in the nurseries under favourable conditions (Chari et al., 1994) and 10-25 per cent to the field crop (Rao and Sitaramaiah, 2001). An eco-friendly alternative to chemical pesticides is the use of bio-pesticides, which encompasses a broad array of microbial pesticides, bio-chemicals derived from micro-organisms and other natural sources, which confer protection against pest damage. The potential benefits to agriculture and public health programmes through the use of bio-pesticides are considerable. India has a vast potential for bio-pesticides. Bio-pesticides, being target pest specific, are presumed to be relatively safe to non-target organisms including human beings. In India, some of the bio-pesticides like Bt, NPV, neem based pesticides and others have already been registered and are in use (Gupta and Dikshit, 2010). Ramaprasad et al., (2000) advocated the use of Biosap (Bacillus thuringiensis var. kurstaki asporogenic) and Biolep (B. t. var. kurstaki sporogenic) against $S$. litura in tobacco nurseries. SINPV caused 96 per cent mortality of $S$. litura within a period of 10 days at a dosage of $6 \times 10^{8} \mathrm{PIBs} /$ larva (Sajap et al., 2000). Similarly, the sequential spray of biopesticides viz., SINPV $250 \mathrm{LE}\left(1.5 \times 10^{12}\right.$
PIB/ha,Btk@1.0 kg/ha was effective against $S$. litura in tobacco nurseries (Rao and Sitaramaiah, 2001).

\section{Materials and Methods}

\section{Layout and design}

The experiment on relative bioefficay of three biopesticides viz., Spinosad, SINPV and Bt.k. alone and in different combinations against $S$. litura was conducted at Horticulture farm, Rajasthan College of Agriculture, Udaipur during rabi 2012-13 and 2013-14.

The experiment was conducted in the randomized block design (RBD) with ten treatments schedules including control, and each treatment schedule was replicated three time. Each treatment schedule was applied three times at 15 days interval initiating first spray in the last week of December when the pest infestation started viz; 30 December and 28 December during rabi 2012-13 and 201314, respectively (Table 1). The cabbage variety Golden acre was transplanted on viz. 30 November and 28 November during rabi 2012-13 and 2013-14, respectively. The plot size was $3.60 \times 3.60 \mathrm{~m}^{2}$ with row to row and plant to plant spacing of $45 \times 45 \mathrm{~cm}$, respectively.

Bioefficacy of three biopesticides viz.; SlNPV, spinosad and Bt.k. alone and in different combinations (Table 2) was evaluated against $S$. litura. The details of different treatment schedules are as follows:

\section{Management schedule of biopesticides}

Pre-calibrated knap sack sprayer was used for spraying the biopesticides care was taken to check the drift of insecticides, by putting polythene sheet screen around each plot at the time of spraying. In all three sprays were applied, first spray was done during the last 
week of December during both the years and subsequent second and third sprays were applied at 15 days interval.

\section{Observations}

Pretreatment population of Spodoptera litura (Fab.) was recorded 24 hours before the scheduled spray. Post treatment population of S. litura was recorded on 3, 7 and 10 day after each spray, on 10 plants were selected randomly in each plot.

\section{Statistical analysis}

Efficacy of different treatments against the $S$. litura was analyzed by analysis of variance. The population data was corrected by the correction factor for determination of per cent reduction (per cent control) using formula given by Henderson and Tilton (1955) refers it to be modification of Abbott (1925).

\section{Per cent reduction in}

$$
\text { population }=100 \times\left[1-\frac{T_{a} x C_{b}}{T_{b} x C_{a}}\right]
$$

Where,

$\mathrm{T}_{\mathrm{a}} \quad=$ Number of insects in different treatments after spray

$\mathrm{T}_{\mathrm{b}} \quad=$ Number of insects in different treatments before spray

$\mathrm{C}_{\mathrm{a}} \quad=$ Number of insects in the untreated check after spray

$\mathrm{C}_{\mathrm{b}} \quad=$ Number of insects in the untreated check before spray

The per cent reduction figures were transformed into arc sine values and subjected to analysis of variance.

\section{Crop yield and economics}

Healthy cabbage heads were harvested when they reached appropriate marketable size and their weight from each treatment was expressed as marketable yield in quintal per hectare and subjected to analysis of variance The avoidable loss and increase in yield of cabbage heads over control were calculated for each treatment by the formula given by Pradhan (1964):

Increase in yield $(\%)=$

Yield in treatment - yield in control

Yield in control

$\mathrm{B}: \mathrm{C}$ ratio over Control $=$

Return in treatment (Rs./ha)

Return in control (Rs./ha) + Cost of insecticides and Labour (Rs./ha)

\section{Results and Discussion}

\section{Effect of biopesticides on the reduction in S. litura population}

In the present investigations, based on the mean per cent reduction in larval population of $S$. litura the results showed that $\mathrm{T}_{1}$ (Spinosad 45 SC @200g/ha - Spinosad 45 SC @200g/ha - Spinosad 45 SC @200g/ha)was most effective in reducing the $S$. litura population upto (70.32\% and $72.13 \%$ ) during rabi 2012-13 and 2013-14, on cabbage crop which was followed by $\mathrm{T}_{6}$ (SINPV 250LE @ 250ml/ha - Spinosad 45 SC @ 200g/haSpinosad 45 SC @ 200g/ha) and resulted in 66.77 and 68.24 per cet reduction during rabi 2012-13 and 2013-14. The present results are in close agreement with the findings of Gupta (2000), Paliwal and Oommen (2005), Stanley et al., (2006) and Topagi et al., (2010) who reported that spinosad suppressed population of S. litura. Mutkule et al., (2009) reported that application of spinosad was superior in suppressing the larval population of S. litura infesting groundnut. SINPV + Spinosad was found against $S$. litura on cabbage reported by Khattab (2005) found SINPV + Spinosad effective against $S$. litura on cabbage. 
The data further revealed that the biopesticides treatment $\mathrm{T}_{3}[B t . k$. (Dipel $8 \mathrm{~L}$ ) at 1lit/ha -Bt.k. (Dipel 8L) at 1lit/ha- Bt.k. (Dipel $8 \mathrm{~L})$ at 1 lit/ha] was least effective against $S$. litura which gave $(56.09 \%$ and $55.24 \%)$ larval population reduction during rabi 201213 and 2013-14. Downard (2004) and Prasad and Ahmed (2009) reported that spinosad was highly effective against $S$. litura, similarly Pokharkar et al., (2001) reported that SINPV and Bacillus thuringiensis was most effective resulting in maximum larval mortality and it was at par with SINPV and B. thuringiensis. Ramegowda and Basavanagoud (2001) and Rao and Sitaramaiah (2001), Hussain et al., (2003) evaluated the efficacy of SINPV and Bt.k. that caused significant reduction in $S$. litura population. Jat and Bhardwaj (2005) reported that Bt.k. and SINPV was most effective against $S$. litura larval population.

Table.1 Details of the treatments and their dose used for the management of Spodoptera litura $(\mathrm{Fab})$

\begin{tabular}{|l|l|l|l|l|}
\hline S. No & Treatments & No. of spray & Formulations & $\begin{array}{l}\text { Quantity/dosages(g or } \\
\mathbf{m l} / \mathbf{h a})\end{array}$ \\
\hline 1. & Spinosad & 3 & $45 \mathrm{SC}$ & 200 \\
\hline 2 & $S l N P V$ & 3 & $250 \mathrm{LE}$ & 250 \\
\hline 3 & $B t . k$. & 3 & $8 \mathrm{~L}$ & 1000 \\
\hline
\end{tabular}

SINPV = Spodoptera litura Nuclear Polyhedrosis Viruses; Bt.k. $=$ Bacillus thuringiensis var. kurstaki

Table.2 Management schedule of biopesticides

\begin{tabular}{|c|c|}
\hline Treatment & Spray of management schedule \\
\hline $\mathrm{T}_{1}$ & $\begin{array}{l}\text { Three spray of spinosad } 45 \mathrm{SC} \text { first at initiation of the pest and subsequent second } \\
\text { and third spray was applied at } 15 \text { days interval. }\end{array}$ \\
\hline $\mathrm{T}_{2}$ & $\begin{array}{l}\text { Three spray of SlNPV } 250 \mathrm{LE} \text { first at initiation of the pest and subsequent second } \\
\text { and third spray was applied at } 15 \text { days interval. }\end{array}$ \\
\hline $\mathrm{T}_{3}$ & $\begin{array}{l}\text { Three spray of Bt.k. (Dipel 8L) first at initiation of the pest and subsequent second } \\
\text { and third spray was applied at } 15 \text { days interval. }\end{array}$ \\
\hline $\mathrm{T}_{4}$ & $\begin{array}{l}\text { First spray of spinosad } 45 \mathrm{SC} \text { at initiation of the pest and subsequent second and } \\
\text { third spray of SlNPV } 250 \mathrm{LE} \text { was applied at } 15 \text { days interval. }\end{array}$ \\
\hline $\mathrm{T}_{5}$ & $\begin{array}{l}\text { First spray of spinosad } 45 \mathrm{SC} \text { at initiation of the pest and subsequent second and } \\
\text { third spray of Bt.k. (Dipel 8L) was applied at } 15 \text { days interval. }\end{array}$ \\
\hline $\mathrm{T}_{6}$ & $\begin{array}{l}\text { First spray of SINPV } 250 \mathrm{LE} \text { at initiation of the pest and subsequent second and } \\
\text { third spray of spinosad } 45 \mathrm{SC} \text { was applied at } 15 \text { days interval }\end{array}$ \\
\hline $\mathrm{T}_{7}$ & $\begin{array}{l}\text { First spray of } S I N P V 250 \mathrm{LE} \text { at initiation of the pest and subsequent second and } \\
\text { third spray of } B t . k \text {. (Dipel } 8 \mathrm{~L} \text { ) was applied at } 15 \text { days interval. }\end{array}$ \\
\hline $\mathrm{T}_{8}$ & $\begin{array}{l}\text { First spray of } B t . k . \text { (Dipel 8L) at initiation of the pest and subsequent second and } \\
\text { third spray of spinosad } 45 \mathrm{SC} \text { was applied at } 15 \text { days interval. }\end{array}$ \\
\hline $\mathrm{T}_{9}$ & $\begin{array}{l}\text { First spray of Bt.k. (Dipel 8L) at initiation of the pest and subsequent second and } \\
\text { third spray of SINPV } 250 \mathrm{LE} \text { was applied at } 15 \text { days interval. }\end{array}$ \\
\hline $\mathrm{T}_{10}$ & Control \\
\hline
\end{tabular}




\section{Int.J.Curr.Microbiol.App.Sci (2017) 6(6): 1853-1866}

Table.3 Relative efficacy of biopesticides against S. litura on cabbage during rabi 2012-13

\begin{tabular}{|c|c|c|c|c|c|c|c|c|c|c|c|c|c|c|c|}
\hline \multirow[b]{3}{*}{ Treatment } & \multirow{3}{*}{$\begin{array}{l}\text { Dose } \\
\text { (ml/ha } \\
\text { or } \\
\mathrm{g} / \mathrm{ha})\end{array}$} & \multirow{3}{*}{$\begin{array}{c}\text { Pre } \\
\text { treatment } \\
\text { population/ } \\
\text { plant }\end{array}$} & \multicolumn{13}{|c|}{ Mean reduction of $S$. litura population (\%) days after sprays } \\
\hline & & & \multicolumn{4}{|c|}{$1^{\text {st }}$ spray (30 Dec. 2012) } & \multicolumn{4}{|c|}{$2^{\text {nd }}$ spray (14 Jan. 2013) } & \multicolumn{4}{|c|}{$3^{\text {rd }}$ spray (29 Jan. 2013) } & \multirow{2}{*}{$\begin{array}{c}\text { Mean } \\
\text { reduction } \\
\text { in } S \text {. litura } \\
\text { population } \\
(\%)\left(1^{\text {st }}\right. \\
+2^{\text {nd }}+3^{\text {rd }} \\
\text { sprays })\end{array}$} \\
\hline & & & $* 3^{\text {rd }}$ & $7^{\text {th }}$ & $10^{\text {th }}$ & Mean & $3^{\text {rd }}$ & $7^{\text {th }}$ & $10^{\text {th }}$ & Mean & $3^{\text {rd }}$ & $7^{\text {th }}$ & $10^{\text {th }}$ & Mean & \\
\hline $\begin{array}{l}\mathrm{T}_{1} \\
\text { Spinosad }\end{array}$ & $200 \mathrm{~g}$ & 2.33 & $\begin{array}{c}51.55 \\
(61.33)^{* *}\end{array}$ & $\begin{array}{c}55.35 \\
(67.66) \\
\end{array}$ & $\begin{array}{c}52.01 \\
(62.11)\end{array}$ & $\begin{array}{c}52.95 \\
(63.70) \\
\end{array}$ & $\begin{array}{c}53.33 \\
(64.33) \\
\end{array}$ & $\begin{array}{c}57.00 \\
(70.33) \\
\end{array}$ & $\begin{array}{c}54.40 \\
(66.11) \\
\end{array}$ & $\begin{array}{c}54.88 \\
(66.92) \\
\end{array}$ & $\begin{array}{c}61.14 \\
(76.68) \\
\end{array}$ & $\begin{array}{c}68.08 \\
(85.99) \\
\end{array}$ & $\begin{array}{c}62.26 \\
(78.33) \\
\end{array}$ & $\begin{array}{c}63.65 \\
(80.33) \\
\end{array}$ & $\begin{array}{c}57.00 \\
(70.32)\end{array}$ \\
\hline $\begin{array}{l}\mathrm{T}_{2} \\
\text { SINPV }\end{array}$ & $250 \mathrm{ml}$ & 2.66 & $\begin{array}{c}47.68 \\
(54.67) \\
\end{array}$ & $\begin{array}{c}49.80 \\
(58.33) \\
\end{array}$ & $\begin{array}{c}46.72 \\
(52.99) \\
\end{array}$ & $\begin{array}{c}48.04 \\
(55.33) \\
\end{array}$ & $\begin{array}{c}48.64 \\
(56.33) \\
\end{array}$ & $\begin{array}{c}52.74 \\
(63.33) \\
\end{array}$ & $\begin{array}{c}49.22 \\
(57.33) \\
\end{array}$ & $\begin{array}{c}50.18 \\
(58.99) \\
\end{array}$ & $\begin{array}{c}53.94 \\
(65.33) \\
\end{array}$ & $\begin{array}{c}60.77 \\
(76.11) \\
\end{array}$ & $\begin{array}{c}56.86 \\
(70.11) \\
\end{array}$ & $\begin{array}{c}57.11 \\
(70.52) \\
\end{array}$ & $\begin{array}{c}51.72 \\
(61.61) \\
\end{array}$ \\
\hline $\mathrm{T}_{3}$ Bt.K & 1lit & 2.99 & $\begin{array}{c}45.38 \\
(50.67) \\
\end{array}$ & $\begin{array}{c}47.68 \\
(54.66) \\
\end{array}$ & $\begin{array}{c}44.64 \\
(49.38)\end{array}$ & $\begin{array}{c}45.92 \\
(51.57)\end{array}$ & $\begin{array}{c}46.53 \\
(52.67) \\
\end{array}$ & $\begin{array}{c}49.80 \\
(58.33) \\
\end{array}$ & $\begin{array}{c}48.26 \\
(55.67) \\
\end{array}$ & $\begin{array}{c}48.22 \\
(55.56)\end{array}$ & $\begin{array}{c}49.09 \\
(57.11) \\
\end{array}$ & $\begin{array}{c}52.93 \\
(63.66) \\
\end{array}$ & $\begin{array}{c}52.35 \\
(62.66) \\
\end{array}$ & $\begin{array}{c}51.41 \\
(61.14)\end{array}$ & $\begin{array}{c}48.50 \\
(56.09) \\
\end{array}$ \\
\hline $\begin{array}{l}\mathrm{T}_{4} \\
\text { Spinosad- } \\
\text { SiNPV- } \\
\text { SINPV } \\
\end{array}$ & $\begin{array}{l}200 \mathrm{~g}- \\
250 \mathrm{ml}- \\
250 \mathrm{ml}\end{array}$ & 2.66 & $\begin{array}{c}51.37 \\
(61.00)\end{array}$ & $\begin{array}{c}54.95 \\
(67.00)\end{array}$ & $\begin{array}{c}51.75 \\
(61.66)\end{array}$ & $\begin{array}{c}52.65 \\
(63.22)\end{array}$ & $\begin{array}{c}49.80 \\
(58.33)\end{array}$ & $\begin{array}{c}53.34 \\
(64.33)\end{array}$ & $\begin{array}{c}49.80 \\
(58.33)\end{array}$ & $\begin{array}{c}50.94 \\
(60.33)\end{array}$ & $\begin{array}{c}54.14 \\
(65.66)\end{array}$ & $\begin{array}{c}60.92 \\
(76.33)\end{array}$ & $\begin{array}{c}57.01 \\
(70.33)\end{array}$ & $\begin{array}{c}57.26 \\
(70.77)\end{array}$ & $\begin{array}{c}53.60 \\
(64.77)\end{array}$ \\
\hline $\begin{array}{l}\mathrm{T}_{5} \\
\text { Spinosad- } \\
\text { Bt.k-Bt.k }\end{array}$ & $\begin{array}{l}200 g- \\
1 \text { lit- } \\
1 \text { lit }\end{array}$ & 2.33 & $\begin{array}{c}51.43 \\
(61.11)\end{array}$ & $\begin{array}{c}55.15 \\
(67.33)\end{array}$ & $\begin{array}{c}51.95 \\
(62.00)\end{array}$ & $\begin{array}{c}52.81 \\
(63.48)\end{array}$ & $\begin{array}{c}47.49 \\
(54.33)\end{array}$ & $\begin{array}{c}50.58 \\
(59.67)\end{array}$ & $\begin{array}{c}48.51 \\
(56.11)\end{array}$ & $\begin{array}{c}48.85 \\
(56.70)\end{array}$ & $\begin{array}{c}49.22 \\
(57.33)\end{array}$ & $\begin{array}{c}53.54 \\
(64.67)\end{array}$ & $\begin{array}{c}52.74 \\
(63.33)\end{array}$ & $\begin{array}{c}51.83 \\
(61.78)\end{array}$ & $\begin{array}{c}51.16 \\
(60.65)\end{array}$ \\
\hline $\begin{array}{l}\mathrm{T}_{6} \text { SINPV- } \\
\text { Spinosad- } \\
\text { Spinosad }\end{array}$ & $\begin{array}{l}250 \mathrm{ml}- \\
200 \mathrm{~g}- \\
200 \mathrm{~g}\end{array}$ & 2.66 & $\begin{array}{c}47.68 \\
(54.66)\end{array}$ & $\begin{array}{c}49.61 \\
(58.00)\end{array}$ & $\begin{array}{c}46.53 \\
(52.67)\end{array}$ & $\begin{array}{c}47.93 \\
(55.11)\end{array}$ & $\begin{array}{c}52.53 \\
(62.99)\end{array}$ & $\begin{array}{c}55.97 \\
(68.66)\end{array}$ & $\begin{array}{c}54.34 \\
(65.99)\end{array}$ & $\begin{array}{c}54.27 \\
(65.88)\end{array}$ & $\begin{array}{c}60.23 \\
(75.33)\end{array}$ & $\begin{array}{c}67.25 \\
(85.00)\end{array}$ & $\begin{array}{c}61.80 \\
(77.66)\end{array}$ & $\begin{array}{c}62.94 \\
(79.33)\end{array}$ & $\begin{array}{c}54.80 \\
(66.77)\end{array}$ \\
\hline $\begin{array}{l}\mathrm{T}_{7} \text { SINPV- } \\
\text { Bt.K-Bt.K }\end{array}$ & $\begin{array}{l}250 \mathrm{ml}- \\
1 \text { lit- } \\
1 \text { lit }\end{array}$ & 2.99 & $\begin{array}{c}47.49 \\
(54.33)\end{array}$ & $\begin{array}{c}49.60 \\
(57.99)\end{array}$ & $\begin{array}{c}46.53 \\
(52.66)\end{array}$ & $\begin{array}{c}47.86 \\
(54.99)\end{array}$ & $\begin{array}{c}46.72 \\
(53.00)\end{array}$ & $\begin{array}{c}50.18 \\
(58.99)\end{array}$ & $\begin{array}{c}48.44 \\
(55.99)\end{array}$ & $\begin{array}{c}48.45 \\
(55.99)\end{array}$ & $\begin{array}{c}49.22 \\
(57.33)\end{array}$ & $\begin{array}{c}53.13 \\
(63.99)\end{array}$ & $\begin{array}{c}52.53 \\
(62.99)\end{array}$ & $\begin{array}{c}51.59 \\
(61.44)\end{array}$ & $\begin{array}{l}49.30 \\
(57.47)\end{array}$ \\
\hline $\begin{array}{l}T_{8} \text { Bt.K- } \\
\text { Spinosad- } \\
\text { Spinosad }\end{array}$ & $\begin{array}{l}\text { 1lit- } \\
200 \mathrm{~g}- \\
200 \mathrm{~g}\end{array}$ & 2.66 & $\begin{array}{c}45.19 \\
(50.33)\end{array}$ & $\begin{array}{c}47.36 \\
(54.11)\end{array}$ & $\begin{array}{c}44.49 \\
(49.11)\end{array}$ & $\begin{array}{c}45.67 \\
(51.18)\end{array}$ & $\begin{array}{c}52.66 \\
(62.66)\end{array}$ & $\begin{array}{c}55.65 \\
(68.11)\end{array}$ & $\begin{array}{c}53.82 \\
(65.11)\end{array}$ & $\begin{array}{c}53.91 \\
(65.29)\end{array}$ & $\begin{array}{c}60.08 \\
(75.11)\end{array}$ & $\begin{array}{c}67.24 \\
(84.99)\end{array}$ & $\begin{array}{c}61.58 \\
(77.33)\end{array}$ & $\begin{array}{c}62.80 \\
(79.14)\end{array}$ & $\begin{array}{c}53.86 \\
(65.21)\end{array}$ \\
\hline $\begin{array}{l}\mathrm{T}_{9} \mathrm{Bt} . \mathrm{K}- \\
\text { SINPV - } \\
\text { SINPV }\end{array}$ & $\begin{array}{l}1 \text { lit- } \\
250 \mathrm{ml}- \\
250 \mathrm{ml} \\
\end{array}$ & 2.33 & $\begin{array}{c}45.39 \\
(50.67)\end{array}$ & $\begin{array}{c}47.48 \\
(54.33)\end{array}$ & $\begin{array}{c}44.42 \\
(48.99)\end{array}$ & $\begin{array}{c}45.70 \\
(51.33)\end{array}$ & $\begin{array}{c}56.67 \\
(48.84)\end{array}$ & $\begin{array}{c}53.08 \\
(63.90)\end{array}$ & $\begin{array}{c}49.67 \\
(58.11)\end{array}$ & $\begin{array}{c}50.52 \\
(59.56)\end{array}$ & $\begin{array}{c}53.75 \\
(64.99)\end{array}$ & $\begin{array}{c}60.67 \\
(75.99)\end{array}$ & $\begin{array}{c}56.82 \\
(69.99)\end{array}$ & $\begin{array}{c}56.99 \\
(70.32)\end{array}$ & $\begin{array}{c}51.01 \\
(60.40)\end{array}$ \\
\hline $\mathrm{T}_{10}$ Control & - & 2.99 & - & - & - & - & - & - & - & & - & - & - & & - \\
\hline S.Em. \pm & & 0.13 & 0.95 & 0.88 & 0.87 & 0.79 & 0.97 & 0.95 & 0.90 & 0.87 & 0.98 & 0.97 & 0.92 & 1.02 & 0.91 \\
\hline C.D at $5 \%$ & & 0.38 & 2.84 & 2.64 & 2.6 & 2.36 & 2.92 & 2.84 & 2.69 & 2.59 & 2.93 & 2.91 & 2.75 & 3.05 & 2.92 \\
\hline
\end{tabular}

**Figures in parentheses are retransformed per cent values, * Days after spray 
Table.4 Relative efficacy of biopesticeds against S. litura on cabbage during rabi 2013-14

\begin{tabular}{|c|c|c|c|c|c|c|c|c|c|c|c|c|c|c|c|}
\hline \multirow{3}{*}{ Treatment } & \multirow{3}{*}{$\begin{array}{c}\text { Dose } \\
\text { (ml/ha } \\
\text { or g/ha) }\end{array}$} & \multirow{3}{*}{$\begin{array}{c}\text { Pre } \\
\text { treatment } \\
\text { population/ } \\
\text { plant }\end{array}$} & \multicolumn{12}{|c|}{ Mean reduction of $S$. litura population (\%) days after sprays } & \multirow{3}{*}{$\begin{array}{c}\text { Mean } \\
\text { reduction } \\
\text { in } S . l i t u r a \\
\text { population } \\
(\%)(1 \mathrm{st} \\
+2 \mathrm{nd}+3 \mathrm{rd} \\
\text { spray })\end{array}$} \\
\hline & & & \multicolumn{4}{|c|}{ 1st spray (28 Dec. 2012) } & \multicolumn{4}{|c|}{ 2nd spray (12 Jan. 2013) } & \multicolumn{4}{|c|}{ 3rd spray (27 Jan. 2013) } & \\
\hline & & & $* 3^{\text {rd }}$ & $7^{\text {th }}$ & $10^{\text {th }}$ & Mean & $3^{\text {rd }}$ & $7^{\text {th }}$ & $10^{\text {th }}$ & Mean & $3^{\text {rd }}$ & $7^{\text {th }}$ & $10^{\text {th }}$ & Mean & \\
\hline $\begin{array}{l}\text { T1 } \\
\text { Spinosad }\end{array}$ & $200 \mathrm{~g}$ & 2.66 & $\begin{array}{c}52.60 \\
(63.11)^{* *}\end{array}$ & $\begin{array}{c}56.17 \\
(69.00)\end{array}$ & $\begin{array}{c}53.33 \\
(64.33)\end{array}$ & $\begin{array}{c}54.01 \\
(65.48)\end{array}$ & $\begin{array}{c}54.40 \\
(66.11)\end{array}$ & $\begin{array}{c}58.49 \\
(72.66)\end{array}$ & $\begin{array}{c}55.76 \\
(68.33)\end{array}$ & $\begin{array}{c}56.18 \\
(69.03)\end{array}$ & $\begin{array}{c}62.28 \\
(78.33)\end{array}$ & $\begin{array}{c}69.51 \\
(87.66)\end{array}$ & $\begin{array}{c}63.22 \\
(76.66)\end{array}$ & $\begin{array}{c}64.05 \\
(80.88)\end{array}$ & $\begin{array}{c}58.15 \\
(72.13)\end{array}$ \\
\hline T2 SINPV & $250 \mathrm{ml}$ & 2.99 & $\begin{array}{c}48.44 \\
(55.99)\end{array}$ & $\begin{array}{c}50.57 \\
(59.66)\end{array}$ & $\begin{array}{c}47.30 \\
(54.00)\end{array}$ & $\begin{array}{c}48.76 \\
(56.55)\end{array}$ & $\begin{array}{c}49.41 \\
(57.66)\end{array}$ & $\begin{array}{c}53.73 \\
(64.99)\end{array}$ & $\begin{array}{c}49.99 \\
(58.66)\end{array}$ & $\begin{array}{c}51.02 \\
(60.44)\end{array}$ & $\begin{array}{c}54.74 \\
(66.66)\end{array}$ & $\begin{array}{c}61.82 \\
(77.67)\end{array}$ & $\begin{array}{c}57.64 \\
(71.33)\end{array}$ & $\begin{array}{c}57.97 \\
(71.89)\end{array}$ & $\begin{array}{c}52.51 \\
(62.96)\end{array}$ \\
\hline T3 Bt.K & 1lit & 3.33 & $\begin{array}{c}44.99 \\
(49.99)\end{array}$ & $\begin{array}{c}46.79 \\
(53.11)\end{array}$ & $\begin{array}{c}44.49 \\
(49.11)\end{array}$ & $\begin{array}{c}45.42 \\
(50.74)\end{array}$ & $\begin{array}{c}45.95 \\
(51.66)\end{array}$ & $\begin{array}{c}49.60 \\
(57.99)\end{array}$ & $\begin{array}{c}47.49 \\
(54.33)\end{array}$ & $\begin{array}{c}47.67 \\
(54.66)\end{array}$ & $\begin{array}{c}48.64 \\
(56.33)\end{array}$ & $\begin{array}{c}52.53 \\
(62.99)\end{array}$ & $\begin{array}{c}51.75 \\
(61.66)\end{array}$ & $\begin{array}{c}50.96 \\
(60.33) \\
\end{array}$ & $\begin{array}{c}48.01 \\
(55.24)\end{array}$ \\
\hline $\begin{array}{l}\text { T4 } \\
\text { Spinosad- } \\
\text { SINPV- } \\
\text { SINPV }\end{array}$ & $\begin{array}{l}200 \mathrm{~g}- \\
250 \mathrm{ml}- \\
250 \mathrm{ml}\end{array}$ & 2.99 & $\begin{array}{c}52.54 \\
(63.00)\end{array}$ & $\begin{array}{c}56.18 \\
(68.99)\end{array}$ & $\begin{array}{c}53.14 \\
(64.00)\end{array}$ & $\begin{array}{c}53.92 \\
(65.33)\end{array}$ & $\begin{array}{c}50.57 \\
(59.66)\end{array}$ & $\begin{array}{c}53.80 \\
(65.11)\end{array}$ & $\begin{array}{c}50.38 \\
(59.33)\end{array}$ & $\begin{array}{c}51.56 \\
(61.37)\end{array}$ & $\begin{array}{c}55.02 \\
(67.11)\end{array}$ & $\begin{array}{c}62.10 \\
(78.11)\end{array}$ & $\begin{array}{c}58.07 \\
(72.00)\end{array}$ & $\begin{array}{c}58.34 \\
(72.41)\end{array}$ & $\begin{array}{c}54.56 \\
(66.37)\end{array}$ \\
\hline $\begin{array}{l}\text { T5 } \\
\text { Spinosad- } \\
\text { Bt.k-Bt.k }\end{array}$ & $\begin{array}{l}\text { 200g- } \\
\text { 1lit-1lit }\end{array}$ & 2.66 & $\begin{array}{c}52.73 \\
(63.33)\end{array}$ & $\begin{array}{c}56.58 \\
(69.66)\end{array}$ & $\begin{array}{c}53.53 \\
(64.66)\end{array}$ & $\begin{array}{c}54.24 \\
(65.88)\end{array}$ & $\begin{array}{c}47.29 \\
(54.00)\end{array}$ & $\begin{array}{c}49.99 \\
(58.66)\end{array}$ & $\begin{array}{c}47.86 \\
(54.99)\end{array}$ & $\begin{array}{c}48.36 \\
(55.88)\end{array}$ & $\begin{array}{c}49.02 \\
(56.99)\end{array}$ & $\begin{array}{c}52.93 \\
(63.66)\end{array}$ & $\begin{array}{c}52.14 \\
(62.33)\end{array}$ & $\begin{array}{c}51.33 \\
(60.99)\end{array}$ & $\begin{array}{c}51.31 \\
(60.92)\end{array}$ \\
\hline $\begin{array}{l}\text { T6 SINPV- } \\
\text { Spinosad- } \\
\text { Spinosad } \\
\end{array}$ & $\begin{array}{l}250 \mathrm{ml}- \\
200 \mathrm{~g}- \\
200 \mathrm{~g}\end{array}$ & 2.99 & $\begin{array}{c}48.25 \\
(55.66)\end{array}$ & $\begin{array}{c}50.25 \\
(59.11)\end{array}$ & $\begin{array}{c}47.10 \\
(53.66)\end{array}$ & $\begin{array}{c}48.70 \\
(56.14)\end{array}$ & $\begin{array}{c}53.33 \\
(64.33)\end{array}$ & $\begin{array}{c}57.42 \\
(70.99)\end{array}$ & $\begin{array}{c}53.35 \\
(67.66)\end{array}$ & $\begin{array}{c}55.40 \\
(67.66)\end{array}$ & $\begin{array}{c}61.82 \\
(77.66)\end{array}$ & $\begin{array}{c}69.05 \\
(87.11)\end{array}$ & $\begin{array}{c}62.05 \\
(77.99)\end{array}$ & $\begin{array}{c}64.10 \\
(80.92)\end{array}$ & $\begin{array}{c}55.71 \\
(68.24)\end{array}$ \\
\hline $\begin{array}{l}\text { T7 SINPV- } \\
\text { Bt.K-Bt.K }\end{array}$ & $\begin{array}{l}250 \mathrm{ml}- \\
1 \text { lit-1lit }\end{array}$ & 3.33 & $\begin{array}{c}48.06 \\
(55.33)\end{array}$ & $\begin{array}{c}50.18 \\
(58.99) \\
\end{array}$ & $\begin{array}{c}46.91 \\
(53.33)\end{array}$ & $\begin{array}{c}48.36 \\
(55.88) \\
\end{array}$ & $\begin{array}{c}46.21 \\
(52.11)\end{array}$ & $\begin{array}{c}49.67 \\
(58.11)\end{array}$ & $\begin{array}{c}47.68 \\
(54.66)\end{array}$ & $\begin{array}{c}47.84 \\
(54.96)\end{array}$ & $\begin{array}{c}49.02 \\
(56.99)\end{array}$ & $\begin{array}{c}52.74 \\
(63.33) \\
\end{array}$ & $\begin{array}{c}52.14 \\
(62.33) \\
\end{array}$ & $\begin{array}{c}51.27 \\
(60.88) \\
\end{array}$ & $\begin{array}{c}49.17 \\
(57.24) \\
\end{array}$ \\
\hline $\begin{array}{l}\text { T8 Bt.K- } \\
\text { Spinosad- } \\
\text { Spinosad }\end{array}$ & $\begin{array}{l}\text { 1lit- } \\
200 \mathrm{~g}- \\
200 \mathrm{~g}\end{array}$ & 2.99 & $\begin{array}{c}44.62 \\
(49.33)\end{array}$ & $\begin{array}{c}46.72 \\
(52.99)\end{array}$ & $\begin{array}{c}44.42 \\
(48.99)\end{array}$ & $\begin{array}{c}45.26 \\
(50.44)\end{array}$ & $\begin{array}{c}53.20 \\
(64.11)\end{array}$ & $\begin{array}{c}57.01 \\
(70.33)\end{array}$ & $\begin{array}{c}54.95 \\
(67.00)\end{array}$ & $\begin{array}{c}55.03 \\
(67.15)\end{array}$ & $\begin{array}{c}61.60 \\
(77.33)\end{array}$ & $\begin{array}{c}68.59 \\
(86.66)\end{array}$ & $\begin{array}{c}61.80 \\
(77.66)\end{array}$ & $\begin{array}{c}63.83 \\
(80.55)\end{array}$ & $\begin{array}{c}54.36 \\
(66.04)\end{array}$ \\
\hline $\begin{array}{l}\text { T9 Bt.K - } \\
\text { SINPV - } \\
\text { SINPV }\end{array}$ & $\begin{array}{l}1 \text { lit- } \\
250 \mathrm{ml}- \\
250 \mathrm{ml} \\
\end{array}$ & 2.66 & $\begin{array}{c}44.81 \\
(49.66)\end{array}$ & $\begin{array}{c}46.72 \\
(53.00)\end{array}$ & $\begin{array}{c}44.43 \\
(49.00)\end{array}$ & $\begin{array}{c}45.32 \\
(50.55)\end{array}$ & $\begin{array}{c}49.67 \\
(58.11)\end{array}$ & $\begin{array}{c}53.75 \\
(65.00)\end{array}$ & $\begin{array}{c}50.19 \\
(58.99)\end{array}$ & $\begin{array}{c}51.18 \\
(60.70)\end{array}$ & $\begin{array}{c}54.95 \\
(67.00)\end{array}$ & $\begin{array}{c}62.03 \\
(78.00)\end{array}$ & $\begin{array}{c}57.86 \\
(71.66)\end{array}$ & $\begin{array}{c}58.60 \\
(72.22)\end{array}$ & $\begin{array}{c}51.45 \\
(61.16)\end{array}$ \\
\hline $\begin{array}{l}\text { T10 } \\
\text { Control }\end{array}$ & - & 2.66 & 0.00 & 0.00 & 0.00 & - & 0.00 & 0.00 & 0.00 & - & 0.00 & 0.00 & 0.00 & - & 0.00 \\
\hline S.Em. \pm & & 0.29 & 0.75 & 0.82 & 0.74 & 0.80 & 0.80 & 0.92 & 0.83 & 0.88 & 0.94 & 0.89 & 0.95 & 1.04 & 0.81 \\
\hline C.D. at 5\% & & 0.54 & 2.24 & 2.46 & 2.23 & 2.38 & 2.41 & 2.77 & 2.50 & 2.63 & 2.82 & 2.67 & 2.86 & 3.10 & 2.43 \\
\hline
\end{tabular}

**Figures in parentheses are retransformed per cent values, * Days after spray 
Table.5 Cumulative efficacy of biopesticides against S. litura on cabbage during rabi 2012-13 and 2013-14

\begin{tabular}{|c|c|c|c|c|c|c|c|}
\hline \multirow[t]{3}{*}{ Treatment } & \multirow{3}{*}{$\begin{array}{c}\text { Dose (ml/ha or } \\
\text { g/ha) }\end{array}$} & \multicolumn{6}{|c|}{ Mean reduction of $S$. litura population (\%) days after sprays } \\
\hline & & \multicolumn{3}{|c|}{ 2012-13 } & \multicolumn{3}{|c|}{ 2013-14 } \\
\hline & & $* 3^{\text {rd }}$ & $7^{\text {th }}$ & $10^{\text {th }}$ & $3^{\text {rd }}$ & $7^{\text {th }}$ & $\mathbf{1 0}^{\text {th }}$ \\
\hline $\mathbf{T}_{1}$ Spinosad & $200 \mathrm{~g}$ & $\begin{array}{c}55.21 \\
(67.44)^{* *}\end{array}$ & $\begin{array}{c}59.77 \\
(74.66)\end{array}$ & $\begin{array}{c}56.07 \\
(68.85)\end{array}$ & $\begin{array}{c}56.28 \\
(69.18)\end{array}$ & $\begin{array}{c}60.96 \\
(76.44)\end{array}$ & $\begin{array}{c}56.64 \\
(69.77)\end{array}$ \\
\hline $\mathbf{T}_{2}$ SINPV & $250 \mathrm{ml}$ & $\begin{array}{c}50.05 \\
(58.77)\end{array}$ & $\begin{array}{c}54.28 \\
(65.92)\end{array}$ & $\begin{array}{c}50.85 \\
(60.14)\end{array}$ & $\begin{array}{c}50.83 \\
(60.10)\end{array}$ & $\begin{array}{c}55.21 \\
(67.44)\end{array}$ & $\begin{array}{c}51.55 \\
(61.33)\end{array}$ \\
\hline T $\mathbf{3}$ Bt.K. & 1lit & $\begin{array}{c}47.16 \\
(53.48)\end{array}$ & $\begin{array}{c}50.11 \\
(58.88)\end{array}$ & $\begin{array}{c}48.39 \\
(55.90)\end{array}$ & $\begin{array}{c}46.52 \\
(52.66)\end{array}$ & $\begin{array}{c}49.62 \\
(58.03)\end{array}$ & $\begin{array}{c}47.89 \\
(55.03)\end{array}$ \\
\hline $\mathrm{T}_{4}$ Spinosad- SINPV-SINPV & $200 \mathrm{~g}-250 \mathrm{ml}-250 \mathrm{ml}$ & $\begin{array}{c}51.74 \\
(61.66)\end{array}$ & $\begin{array}{c}56.30 \\
(69.22)\end{array}$ & $\begin{array}{c}52.80 \\
(63.44)\end{array}$ & $\begin{array}{c}52.68 \\
(63.25)\end{array}$ & $\begin{array}{c}57.25 \\
(70.73)\end{array}$ & $\begin{array}{c}53.79 \\
(65.11)\end{array}$ \\
\hline$T_{5}$ Spinosad- Bt.k.-Bt.k. & 200g-1lit-1lit & $\begin{array}{c}49.36 \\
(57.59)\end{array}$ & $\begin{array}{c}53.06 \\
(63.89)\end{array}$ & $\begin{array}{c}51.05 \\
(60.48)\end{array}$ & $\begin{array}{c}49.66 \\
(58.10)\end{array}$ & $\begin{array}{c}53.12 \\
(63.99)\end{array}$ & $\begin{array}{c}51.15 \\
(60.66)\end{array}$ \\
\hline T $_{6}$ SINPV-Spinosa-Spinosad & $250 \mathrm{ml}-200 \mathrm{~g}-200 \mathrm{~g}$ & $\begin{array}{c}53.32 \\
(64.32)\end{array}$ & $\begin{array}{c}57.13 \\
(70.55)\end{array}$ & $\begin{array}{c}53.99 \\
(65.44)\end{array}$ & $\begin{array}{c}54.23 \\
(65.83) \\
\end{array}$ & $\begin{array}{c}58.30 \\
(72.40)\end{array}$ & $\begin{array}{c}54.59 \\
(66.43) \\
\end{array}$ \\
\hline T $_{7}$ SINPV- Bt.K.-Bt.K. & 250ml-1lit-1lit & $\begin{array}{c}47.80 \\
(54.88)\end{array}$ & $\begin{array}{c}50.95 \\
(60.32)\end{array}$ & $\begin{array}{c}49.14 \\
(57.21)\end{array}$ & $\begin{array}{c}47.76 \\
(54.81)\end{array}$ & $\begin{array}{c}50.85 \\
(60.14)\end{array}$ & $\begin{array}{c}48.89 \\
(56.77)\end{array}$ \\
\hline$T_{8}$ Bt.K.-Spinosad-Spinosad & 1lit-200g-200g & $\begin{array}{c}52.36 \\
(62.70) \\
\end{array}$ & $\begin{array}{c}56.21 \\
(69.07) \\
\end{array}$ & $\begin{array}{c}53.04 \\
(63.85) \\
\end{array}$ & $\begin{array}{c}52.88 \\
(63.59) \\
\end{array}$ & $\begin{array}{c}56.78 \\
(69.99) \\
\end{array}$ & $\begin{array}{c}53.45 \\
(64.55) \\
\end{array}$ \\
\hline T9 Bt.K. -SINPV -SINPV & 1lit-250ml-250ml & $\begin{array}{c}47.77 \\
(54.83)\end{array}$ & $\begin{array}{c}53.57 \\
(64.74)\end{array}$ & $\begin{array}{c}50.20 \\
(59.03)\end{array}$ & $\begin{array}{c}49.75 \\
(58.25)\end{array}$ & $\begin{array}{c}53.93 \\
(65.33)\end{array}$ & $\begin{array}{c}50.69 \\
(59.88)\end{array}$ \\
\hline$T_{10}$ Control & - & 0.00 & 0.00 & 0.00 & 0.00 & 0.00 & 0.00 \\
\hline S.Em. \pm & - & 0.96 & 0.93 & 0.89 & 0.83 & 0.87 & 0.84 \\
\hline C.D at $5 \%$ & - & 2.55 & 2.79 & 2.68 & 2.48 & 2.63 & 2.66 \\
\hline
\end{tabular}

**Figures in parentheses are retransformed per cent values, * Days after spray 
Table.6 Comparative economics of biopesticide treatments against S. litura on cabbage during rabi 2012-13

\begin{tabular}{|c|c|c|c|c|c|c|c|c|c|c|c|}
\hline S.No. & Treatments & $\begin{array}{c}\text { Dose } \\
\text { (ml/ha or } \\
\text { g/ha) }\end{array}$ & $\begin{array}{l}\text { No. of } \\
\text { Sprays }\end{array}$ & $\begin{array}{c}\text { Average } \\
\text { yield } \\
\text { (qt/ha) }\end{array}$ & $\begin{array}{l}\text { Increase } \\
\text { in yield } \\
\text { over } \\
\text { control } \\
\text { (qt/ha) } \\
\end{array}$ & $\begin{array}{c}\text { Mean } \\
\text { avoidble } \\
\text { loss }(\%)\end{array}$ & $\begin{array}{c}\text { Gross } \\
\text { Return } \\
\text { (Rs./ha) }\end{array}$ & $\begin{array}{c}\text { Return of } \\
\text { increased } \\
\text { yield over } \\
\text { control } \\
\text { (Rs./ha) } \\
\end{array}$ & $\begin{array}{c}\text { Total } \\
\text { expenditure } \\
\text { (labour + } \\
\text { insecticide) }\end{array}$ & $\begin{array}{c}\text { Net } \\
\text { profit } \\
\text { (Rs./ha) }\end{array}$ & $\begin{array}{c}\text { C:B } \\
\text { Ratio } \\
\text { Return } \\
\text { over } \\
\text { control }\end{array}$ \\
\hline $\mathrm{T}_{1}$ & Spinosad & $\begin{array}{l}200 \mathrm{~g}-200 \mathrm{~g}- \\
200 \mathrm{~g}\end{array}$ & 3 & 287.35 & 91.70 & 00.00 & 431025 & 137550 & 10082 & 127468 & $1: 1.419$ \\
\hline $\mathrm{T}_{2}$ & SINPV & $\begin{array}{l}250 \mathrm{ml}- \\
250 \mathrm{ml}- \\
250 \mathrm{ml}\end{array}$ & 3 & 263.05 & 67.40 & 8.46 & 394575 & 101100 & 2682 & 98418 & $1: 1.332$ \\
\hline $\mathrm{T}_{3}$ & Bt.k & $\begin{array}{l}1 \text { lit-1lit-1 } \\
\text { lit }\end{array}$ & 3 & 240.45 & 44.80 & 16.32 & 360675 & 67200 & 2832 & 64368 & $1: 1.220$ \\
\hline $\mathrm{T}_{4}$ & $\begin{array}{l}\text { Spinosad- } \\
\text { SINPV- } \\
\text { SINPV }\end{array}$ & $\begin{array}{l}200 \mathrm{~g}- \\
250 \mathrm{ml}- \\
250 \mathrm{ml}\end{array}$ & $1-1-1$ & 272.86 & 77.21 & 5.04 & 409290 & 115815 & 5149 & 110666 & $1: 1.370$ \\
\hline $\mathrm{T}_{5}$ & $\begin{array}{l}\text { Spinosad- } \\
\text { Bt.k- Bt.k }\end{array}$ & $\begin{array}{l}\text { 200g-1lit- } \\
1 \text { lit }\end{array}$ & $1-1-1$ & 255.15 & 59.50 & 11.20 & 382725 & 892500 & 5249 & 84001 & $1: 1.281$ \\
\hline $\mathrm{T}_{6}$ & $\begin{array}{l}\text { SlNPV- } \\
\text { Spinosad - } \\
\text { Spinosad } \\
\end{array}$ & $\begin{array}{l}250 \mathrm{ml}- \\
200 \mathrm{~g}-200 \mathrm{~g}\end{array}$ & $1-1-1$ & 279.45 & 83.80 & 2.71 & 419175 & 125700 & 7615 & 118085 & $1: 1.392$ \\
\hline $\mathrm{T}_{7}$ & $\begin{array}{l}\text { SINPV-Bt.k } \\
\text {-Bt.k }\end{array}$ & $\begin{array}{l}250 \mathrm{ml}-1 \text { lit- } \\
1 \text { lit }\end{array}$ & $1-1-1$ & 247.85 & 52.20 & 13.75 & 371775 & 78300 & 2782 & 75518 & $1: 1.255$ \\
\hline $\mathrm{T}_{8}$ & $\begin{array}{l}\text { Bt.k- } \\
\text { Spinosad- } \\
\text { Spinosad }\end{array}$ & $\begin{array}{l}\text { 1lit-200g- } \\
200 \mathrm{~g}\end{array}$ & $1-1-1$ & 267.85 & 72.20 & 6.79 & 401775 & 108300 & 7665 & 100635 & 1:1.334 \\
\hline $\mathrm{T}_{9}$ & $\begin{array}{l}\text { Bt.k- } \\
\text { SINPV- } \\
\text { SINPV }\end{array}$ & $\begin{array}{l}\text { 1lit-250ml- } \\
250 \mathrm{ml}\end{array}$ & $1-1-1$ & 250.25 & 54.60 & 12.82 & 375375 & 81900 & 2732 & 79168 & $1: 1.267$ \\
\hline $\mathrm{T}_{10}$ & Control & - & - & 195.65 & 0.00 & 31.91 & 293475 & - & - & - & - \\
\hline
\end{tabular}

(1) Present price of insecticides: Spinosad Rs. $=1533 / 100 \mathrm{ml}$ or g, SINPV Rs. =600/250LE (250ml), Bt. K Rs. = 650/kg or lit

(2) Labour charge @ 147/- per day per labour (2 labour required/spray/day and 6 labour required for 3 spray)

(3) Sale price of cabbage Rs. $=15 / \mathrm{kg}$ 
Table.7 Comparative economics of biopesticides against S. litura on cabbage during rabi 2013-14

\begin{tabular}{|c|c|c|c|c|c|c|c|c|c|c|c|}
\hline S.No. & Treatments & $\begin{array}{c}\text { Dose } \\
\text { (ml/ha or } \\
\text { g/ha) }\end{array}$ & Sprays & $\begin{array}{c}\text { Average } \\
\text { yield } \\
\text { (qt/ha) }\end{array}$ & $\begin{array}{c}\text { Increase } \\
\text { in yield } \\
\text { over } \\
\text { control } \\
\text { (qt/ha) } \\
\end{array}$ & $\begin{array}{c}\text { Mean } \\
\text { avoidable } \\
\text { loss }(\%)\end{array}$ & $\begin{array}{c}\text { Gross } \\
\text { Return } \\
\text { (Rs./ha) }\end{array}$ & $\begin{array}{c}\text { Return of } \\
\text { increased } \\
\text { yield over } \\
\text { control } \\
\text { (Rs./ha) } \\
\end{array}$ & $\begin{array}{c}\text { Total } \\
\text { expenditure } \\
\text { (labour + } \\
\text { insecticide) }\end{array}$ & $\begin{array}{c}\text { Net } \\
\text { profit } \\
\text { (Rs./ha) }\end{array}$ & $\begin{array}{c}\text { C:B } \\
\text { Ratio } \\
\text { Return } \\
\text { over } \\
\text { control } \\
\end{array}$ \\
\hline $\mathrm{T}_{1}$ & Spinosad & $\begin{array}{l}200 \mathrm{~g}- \\
200 \mathrm{~g}-200 \mathrm{~g}\end{array}$ & 3 & 291.15 & 91.05 & 0.00 & 436725 & 136575 & 10202 & 126373 & $1: 1.407$ \\
\hline $\mathrm{T}_{2}$ & SINPV & $\begin{array}{l}250 \mathrm{ml}- \\
250 \mathrm{ml}- \\
250 \mathrm{ml}\end{array}$ & 3 & 267.10 & 67.00 & 8.26 & 400650 & 100500 & 2802 & 97698 & $1: 1.322$ \\
\hline $\mathrm{T}_{3}$ & Bt.k & $\begin{array}{l}1 \text { lit-1lit-1 } \\
\text { lit }\end{array}$ & 3 & 244.75 & 44.65 & 15.94 & 367125 & 66975 & 2952 & 64023 & $1: 1.211$ \\
\hline $\mathrm{T}_{4}$ & $\begin{array}{l}\text { Spinosad- } \\
\text { SINPV- } \\
\text { SlNPV }\end{array}$ & $\begin{array}{l}200 \mathrm{~g}- \\
250 \mathrm{ml}- \\
250 \mathrm{ml}\end{array}$ & $1-1-1$ & 276.55 & 76.45 & 5.01 & 414825 & 114675 & 5268 & 109407 & $1: 1.358$ \\
\hline $\mathrm{T}_{5}$ & $\begin{array}{l}\text { Spinosad- } \\
\text { Bt.k- Bt.k }\end{array}$ & $\begin{array}{l}\text { 200g-1lit- } \\
11 \text { it }\end{array}$ & $1-1-1$ & 258.85 & 58.75 & 11.09 & 388275 & 88125 & 5369 & 82756 & $1: 1.271$ \\
\hline $\mathrm{T}_{6}$ & $\begin{array}{l}\text { SlNPV- } \\
\text { Spinosad - } \\
\text { Spinosad }\end{array}$ & $\begin{array}{l}250 \mathrm{ml}- \\
200 \mathrm{~g}-200 \mathrm{~g}\end{array}$ & $1-1-1$ & 283.15 & 83.05 & 2.75 & 424725 & 124575 & 7735 & 116840 & 1:1379 \\
\hline $\mathrm{T}_{7}$ & $\begin{array}{l}\text { S1NPV-Bt.k - } \\
\text { Bt.k }\end{array}$ & $\begin{array}{l}250 \mathrm{ml}- \\
1 \text { lit-1lit }\end{array}$ & $1-1-1$ & 251.65 & 51.55 & 13.57 & 377475 & 77325 & 2902 & 74423 & $1: 1.245$ \\
\hline $\mathrm{T}_{8}$ & $\begin{array}{l}\text { Bt.k- } \\
\text { Spinosad- } \\
\text { Spinosad }\end{array}$ & $\begin{array}{l}\text { 1lit-200g- } \\
200 \mathrm{~g}\end{array}$ & $1-1-1$ & 271.10 & 71.00 & 6.70 & 406650 & 106500 & 7785 & 98718 & $1: 1.320$ \\
\hline $\mathrm{T}_{9}$ & $\begin{array}{l}\text { Bt.k-SINPV- } \\
\text { SINPV }\end{array}$ & $\begin{array}{l}\text { 1lit- } \\
250 \mathrm{ml}- \\
250 \mathrm{ml}\end{array}$ & $1-1-1$ & 254.05 & 53.95 & 12.74 & 381075 & 80925 & 2852 & 78073 & $1: 1.258$ \\
\hline $\mathrm{T}_{10}$ & Control & - & - & 200.10 & 00.00 & 31.27 & 300150 & - & - & - & - \\
\hline
\end{tabular}

(1) The present price of insecticides: Spinosad Rs. $=1533 / 100 \mathrm{ml}$ or g, SlNPV Rs. =600/250LE (250ml) Bt.k Rs. = 650/kg or lit

(2) Labour charge @ 167/- per day per labor (2 labour required/spray/day and 6 labour required for 3 spray)

(3) Sale price of cabbage Rs. = $15 / \mathrm{kg}$ 
Mabrouk and Abbas (2002), Basappa and Singh (2003), Kumari and Singh (2009) and Ali et al., (2011) reported that the virulence of SINPV proved most effective against $S$. litura larval population. The results are in conformity with Patil and Hegde (2009) who recorded efficacy of Bt.k. and SINPV and found then most effective against $S$. litura larval population. Mandal et al., (2009) recommended three application of spinosad (Success $2.5 \mathrm{SC}$ ) at 15 and $30 \mathrm{~g}$ a.i. for management of $S$. litura. Muthukumar et al., (2007) reported that spinosad at $75 \mathrm{~g}$ ai $/ \mathrm{h}$, Spinosad, Biolep, emamectin benzoate and neem oil proved safer to natural enemies in the cauliflower ecosystem. Newly introduced insecticides such as spinosad, indoxacarb, $S I N P V$, rimon or corzen showed proven efficacy against S. litura (Gupta et al., 2004; Mohapatra et al., 1995; Pramanik and Chatterjee, 2004; Muthukumar et al., 2007). Bhutia et al., (2012) reported that the virulenceof SINPV proved most effective against $S$. litura larval population. However, Krishnaiah et al., (1981), Malathi et al., (1999), Sharma (2000) and Chatterjee (2008) reported that Bt.k. was effective against $S$. litura larval population. Babu and Krishnayya (1998), reported that the Neem oil, Bt.k. and their combinations were relatively less effective against $S$. litura but were however superior to untreated control.

\section{Effect of biopesticides application on head yield of cabbage}

The results showed that the yield of cabbage heads in all the biopesticidal treatments was significantly superior over untreated plots. The highest yield of 287.35 and $291.15 \mathrm{q} \mathrm{ha}^{-1}$ was obtained from the plots treated with $\mathrm{T}_{1}$ (Spinosad 45 SC @ 200g/ha - Spinosad 45 SC @ 200g/ha - Spinosad 45 SC @200g/ha)and the minimum yield was recorded in $\mathrm{T}_{3}$ [Bt.k. (Dipel 8L) at 1lit/ha - Bt.k. (Dipel 8L) at 1lit/ha - Bt.k. (Dipel 8L) at 1lit/ha], 240.45 and $244.75 \mathrm{q} \mathrm{ha}^{-1}$, during rabi 2012-13 and 2013-14, respectively. The results are in conformity with that of Gupta (2000) who recorded significantly higher yield in spinosad. The present results are also supported from the results of Gupta and Jain (2001) who reported better yield of cabbage heads by the treatment of spinosad. The present results are in agreement with the findings of Prasad and Wadhwani (2005), Kumar and Singh (2009) and Ali et al., (2011) who recorded higher yield of cabbage head obtained from the treatment of SINPV. These findings are in partial agreement with the results of Ashokan et al., (1996) who observed significant increase in yield of cabbage heads from the treatment of Bt.K.

\section{Cumulative efficacy of biopesticides}

The data presented in table 5 reveal that during 2012-13 the treatment schedule comprising three spray of spinosad $45 \mathrm{SC}$ at $200 \mathrm{~g} / \mathrm{ha}$ at 15 days interval was found effective which caused 67.44, 74.66 and 68.85 per cent reduction in larvae population at 3, 7, and 10 days after three spray respectively. It was followed by treatment schedule $\mathrm{T}_{6}$ comprising spray of SINPV at $250 \mathrm{LE} /$ ha followed by two spray of spinosad at $200 \mathrm{~g} / \mathrm{ha}$ which caused $64.32,70.55$ and 65.54 per cent reduction at 3,7 and 10 days after three spray, respectively (Table 5). The data on cumulative bioefficacy further revealed treatment Bt.k. was the least effective among the treatment. Similar results were also recorded in table 6 revealed that during 2013-14 the treatment schedule comprising three spray of spinosad $45 \mathrm{SC}$ at $200 \mathrm{~g} / \mathrm{ha}$ at 15 days interval was found effective which caused 69.18, 76.44 and 69.77 per cent reduction in larvae population at 3, 7, and 10 days after three spray respectively. It was followed by treatment schedule $\mathrm{T}_{6}$ comprising spray of SINPV at $250 \mathrm{LE} / \mathrm{ha}$ followed by two spray of spinosad 
at $200 \mathrm{~g} / \mathrm{ha}$ which caused $65.83,72.40$ and 66.43 per cent reduction at 3,7 , and 10 days after three spray, respectively. Three spray of Bt.k. was the least effective among all the treatment.

\section{Economics of biopesticides treatments}

The utility of any biopesticides in the pest management programme is not only evaluated by its relative potency against the target pest and the period for which its application provides protection to the crop, but the economics of the treatments also is a major consideration. Hence, the benefit cost ratio was also worked out in the present investigation. The data revealed that the maximum profit was obtained from the treatment of $\mathrm{T}_{1}($ Spinosad $45 \mathrm{SC} @ 200 \mathrm{~g} / \mathrm{ha}$ Spinosad 45 SC @ 200 gm/ha - Spinosad 45 SC @200g/ha)which gave a benefit:cost ratio of 1.419 and 1.407, during rabi 2012-13 and 2013-14, respectively. The minimum benefit cost ratio was found in the treatment of $\mathrm{T}_{3}$ [Bt.k. (Dipel 8L) at 1lit/ha - Bt.k. (Dipel 8L) at 1lit/ha - Bt.k. (Dipel 8L) at 1lit/ha] which gave a benefit cost ratio of 1.220 and 1.211 , during rabi 2012-13 and 2013-14, respectively. Contrary to the present findings Pokharkar et al., (2001) reported that treatment of SINPV and Bt.k. to sufficient cost benefit ratio with $1: 22.40$ and 1:20.29, respectively. The present findings are supported by Prasad and Wadhwani (2005) who reported that SINPV gave higher economic return $(\mathrm{C}$ : $\mathrm{B}$ ratio $1: 1.524)$. The benefit cost ratio of biopesticides was comparatively less due to their higher cost (Table 7).

In the present investigations the relative efficacy of three biopesticides viz., spinosad, Bt.k. and SINPV in alone and in nine different schedule combinations was evaluated against S. litura. The data for both years (2012-13 and 2013-14) revealed that the treatment schedule comprising three spray of Spinosad $45 \mathrm{SC}$ at $200 \mathrm{~g} / \mathrm{ha}$ first at initiation of the pest and subsequent second and third spray was applied at 15 days interval was most effective in reducing the $S$. litura larval population upto 70.32 and 72.13 per cent during rabi 2012-13 and 2013-14, respectively (tables 3 and 4), but the minimum reduction of 56.09 and 55.24 per cent was recorded in treatment schedule comprising three spray of Bt.k. (Dipel 8L) at 1lit/ha first at initiation of the pest and subsequent second and third spray was applied at 15 days interval. The highest yield of 287.35 and $291.15 \mathrm{q} \mathrm{ha}^{-1}$ was obtained from the plots treated with treatment schedule comprising three spray of Spinosad $45 \mathrm{SC}$ at $200 \mathrm{~g} / \mathrm{ha}$, whereas, the minimum yield 240.45 and $244.75 \mathrm{q} \mathrm{ha}^{-1}$ was recorded in treatment schedule comprising three spray of Bt.k. (Dipel 8L) at 1lit/ha, during rabi 2012-13 and 2013-14. The increase in yield over untreated control plot was maximum (46.87 and $45.50 \%$ during 2012-13 and 2013-14) in the treatment schedule comprising three spray of spinosad $45 \mathrm{SC}$ at $200 \mathrm{~g} / \mathrm{ha}$; whereas, the lowest increase in yield (22.90 and $22.31 \%$ during 2012-13 and 2013-14) in treatment schedule comprising three spray of $B t . k$. (Dipel 8L) at 1lit/ha. The highest benefit: cost ratio of 1.419 and 1.407 was recorded in the treatment schedule comprising three spray of spinosad $45 \mathrm{SC}$ at $200 \mathrm{~g} / \mathrm{ha}$ and minimum benefit cost ratio of 1.220 and 1.211 was recorded in the treatment schedule comprising three spray of Bt.k. (Dipel 8L) at 1lit/ha during rabi 2012-13 and 2013-14.

\section{References}

Ali, S., Kumar, R., Dohray, R. K. and Kumar S. 2011. Evaluation of SINPV with botanicals for management of Spodoptera litura on cauliflower. Annals of Plant Protection Sciences, 19(2): 464-466.

Ashokan, R., Mohan, K. S. and 
Gopalkrishanan, C. 1996. Effect of commercial formulation of Bacillus thuringiensis on yield of cabbage at Bangalore, Insect Environment, 2 (2): 58-59.

Ayyar, T.V. and Ramakrishna.1963. Pest of vegetables. In hand book of Entomology pests. Entomology in India.

Basappa, H. and Singh, H. 2003. Effect of sequential application of biopesticides and endosulfan on Spodoptera litura (Fab.). Proceeding of National Symposium Frontier Areas of Entomology Research, 5-7 November, New Delhi.

Bhutia, K.C., Chakravarthy, A.K., Doddabasappa, B., Narabenchi, G.B. and Lingaraj, V.K. 2012. Evaluation and production of improved formulation of Nuclear Polyhedrosis Virus of Spodoptera litura. Bulletin of Insectology, 65(2): 247-256.

Chari, M.S., Rao, R.S.N. and Sreedhar, U. 1994. Integrated management of Spodoptera litura (Fab.) in India. Bulletin of Species. CORESTA. Congress, Harare, 99.

Chatterjee, Hirka. 2008. Potentialities of some microbials against important lepidopteran borers in West Bengal. (Insect and Environment VIII). Emerging trends of researches in insect pest management and environmental safety, Volume, 9(1): 181-188.

Choudhari, N., Deb, D.C. and Semapati, S.K. 2001. Assessment of loss in yield caused by pest coplex of tomato under terai region of West Bengal. Research on Crops, 2(1): 71-79.

Choudhari, N., Ghosh, J. and Senapati, S.K. 2001. Incidence of insect pests of cabbage in relation to prevailing climatic conditions of Tarai region. Indian Journal of Entomology, 63(4): 421-428.

Downward, P. 2004. Spinosad controls a range of lepidopteran pests in crucifers in Australia. The management of diamond back moth and other crucifer pests. Proceedings of the Fourth International Workshop, Melbourne, Victoria, Australia, 351-356.

Gupta, G.P., Seema, R., Ajanta, B., and Raghuraman, M. 2004. Relative toxicity of certain new insecticides against Spodoptera litura (Fab.). Pesticides Research Journal, 16: 45-47.

Gupta, H.C.L. 2000. Bio-efficacy of spinosad $2.5 \mathrm{SC}$ against pests of cabbage. Final technical report of the project sponsored by De-Nocil Crop Production Limited.

Maharana Pratap University of Agriculture and Techonology, Udaipur, pp. 1-17.

Gupta, H.C.L. and Jain, K.L. 2001. Bioefficacy of spinosad against pests of cabbage. National Conference: Plant Protection, NCPP-New Horizons in the Millennium Entomological Research Association, Udaipur, Pp-24.

Gupta, Suman and Dikshit, A.K. 2010. Biopesticides: An ecofriendly approach for pest control. Journal of Biopesticides, 3(1): 186-18.

Hussain, M.A., Pachori, R. and Choudhary, B.S. 2003. Management of Spodoptera litura (Fab.) on cabbage with special reference to microbial pesticides. Research on Crop, 4(2): 263-267.

Jahan, F., Askarianzadeh, A., Abbasipour, H., Hasanshahi, G. and Saeedizadeh. 2013. Effect of various cauliflower cultivars on population density fluctuations of the cabbage aphid, Brevicoryne brassicae (L.) (Homptera: Aphididae) and its parasitoid Diaeretiella rapae (McIntosh) (Hymenoptera: Braconidae). Archives of Phytopathology and Plant Protection, 46(18): 2208.

Jat, M.C. and Bhardwaj, S.C. 2005. Combined effect of Bacillus thuringiensis and SINPV with 
malathion, decamethrin and azadirachtin against larvae of Spodoptera litura (Fabricius) on cauliflower. Annals of Plant Protection Sciences, 13(1): 119-122.

Khattab, M. 2007. Enhancement of the cotton leaf worm, Spodoptera littoralis (Lepidoptera: Noctuidae) Nuclear Polyhedrosise Virus activity by Spinosad. Egyptian Journal of Biological Pest Control, 17(1/2): 147152.

Krishnaiah, K., Mohan, N.J. and Prasad, V.G. 1981. Efficacy of Bacillus thuringiensis for the control of lepidopterous pests of vegetable crops. Entomon, 6(2): 87-94.

Kumari, V. and Singh, N.P. 2009. Spodoptera litura Nuclear Polyhedrosis Virus (SINPV) as a component in Integrated Pest Management (IPM) of Spodoptera litura (Fab.) on cabbage. Journal of Biopesticides, 2(1): 84-86. 20.

Lall, B.S. 1964. Vegetable pests. In Entomology in India. Entomological Society of India, New Delhi.187-211.

Mabrouk, A.M. and Abbas, E.P. 2002. Effectiveness of nuclear polyhedrosis virus against the cotton leafworm, Spodoptera littoralis (Biosd.) (Lepidoptera: Noctuidae) ArabUniversities. Journal of Agricultural Science, 10(1): 391-403.

Malathi, S., Sriramulu, M. and Babu, T. Ramesh. 1999. Evaluation of certain eco-friendly insecticides against Lepidoptera pests of cabbage. Indian Journal of Entomology, 16 (2): 127133.

Mandal, K., Gagan, J., and Singh, B. 2009. Dissipation kinetics of spinosad on cauliflower (Brassica oleraceavar. Botrytis L.) Under subtropical conditions of Punjab, India. Bulletin of Environmental Contamination and Toxicology, 83(6): 808-811.

Mohapatra, S., Sawarkar, S., Patnaik, H.P. and Senapati, B. 1995. Antifeedant activity of solvent extracts of neem seed kernel against Spodoptera litura (Fab.) and their persistency against sunlight through encapsulation. International journal of pest management, 41(3): 154156.

Muthukumar, M., Sharma, R. K., and Sinha, S. R. (2007). Field efficacy of biopesticides and new insecticides against major insect pests and their effect on natural enemies in cauliflower. Pesticide Research Journal, 19 (2): 190-196.

Mutkule, D.S., Patil, B.V., Aglave, B.N.and Jagtap, P.K. 2009. Field evaluation of new molecules for the control of Spodoptera litura (Fabricius) on groundnut. Indian Journal of Entomology, 71(4): 292-295.

Paliwal, S. and Oommen, S. 2005. Effect of spinosad against tobacco caterpillar in cauliflower. Indian Journal of Applied Entomoloy, 10(2): 116-117.

Patil, R.H. and Hegde. 2009. Biorational approaches for the management of Lepidopteran pests of soybean. Karnataka Journal of Agricultural Sciences, 22(3): 484-486.

Pokharkar, D.S., Charati, S.N., Bade, B.A. and Ghorpsde, S.A. 2001. Evaluation of biocontrol agents for managing Spodoptera litura (Fab.) damaging potato (Solanum tuberosum). Indian Journal of Agricultural Science, 71(12): 797-799.

Pradhan, S. 1964. Assessment of losses caused by insect-pests of crops of crops and estimation of insect population. In: Entomology in India, Entomological society of India, New Delhi, pp. 17-58.

Pramanik, P and Chatterjee, M.L. 2004. Effects of novaluron on the population of Plutella xylostella and Spodoptera litura (Fab.) on cabbage. Annals of Plant Protection Sciences, 12(1): 204- 
205.

Prasad, A. and Yogita, Wadhwani. 2005. A. thesis submitted to Mohan Lal Sukhadia University, Udaipur Rajasthan (Indian).

Prasad, N.V.V.S.D. and Ahmed, Khalid. 2009. Efficacy of spinosad 45 SC against thrips, Scitrothrips dorsalis (Hood) and pod borer, Spodoptera exigua (Hubner) on chillies. Pesticide Research Journal, 21(1): 49-51.

Ramaprasad, G., Sitaramaiah, S. and Sreedhar, U. 2000. Efficay of Bacillus thuringiensis Berliner formulations against tobacco caterpillar, Spodoptera litura (Fab.) in tobacco nurseries. Tobacco Research., 26 (2): 1-6.

Ramegowde, G.K. and Basavangoud, K. 2001. Management of insecticides resistance in Spodoptera litura (Fab.) by microbial agents and newer molecule. Karnataka Journal of Science, 14(4): 1088-1092.

Rao, S.G. and Sitaramaiah, S. 2001. Application strategies for microbial pesticides against Spodoptera litura (Fab.) and Helicoverpa armigera (Hub.). Tobacco Research, 27(1): 1218.

Rao, S.R.K. and Lal, O.P. 2005. Seasonal incidence of mustard aphid, Lipaphis erysimi (Kalt.) and diamond back moth, Plutella xylostella (L.) on cabbage. Journal of Insect Science, 18(2): 106110.

Sajap. A.S., Kotuli, J.R., Bakir, M.A., Hong, L.W., Samad, N.A. and Kadir, H.A. 2000. Pathogenicity and characteristics of Spodoptera litura Nucleopolyhedrovirus from Peninsular Malaysia. Pertanika Journal of Tropical Agriculture Science, 23(1): 23-28.

Sharma, A.N. 2000. Bioefficacy of Bacillus thuringensis based biopesticides against Spodoptera litura (Fab.) and Spilaractia oblique Walker feeding on soybean. Crop Research. Hisar, 19(2): 373-375.

Stanley, J., Chandrasekaran, S., Regupathy, A. and Jasmine, R.S. 2006. Base line toxicity of emamectin and spinosad to Spodoptera litura (Fab.). Annals of Plant Protection Sciences, 14(2): 346349.

Topagi, S.C., Rao, G.R.., Rao, P.A. and Rao, V.S. 2010. Evaluation of certain ecofriendly insecticides against insect-pest complex of sunflower, Helianthus annuus L. Journal of Oilseeds Research, 27(1): 67-68.

\section{How to cite this article:}

Jat, G.C., R. Swaminathan, P.C. Yadav, Swati, H.L. Deshwal, Suman Choudhary and Suresh Kumar Yadav. 2017. Relative Efficacy and Economics of Bio-pesticides against Spodoptera litura (Fab.) on Cabbage. Int.J.Curr.Microbiol.App.Sci. 6(6): 1853-1866.

doi: https://doi.org/10.20546/ijcmas.2017.606.216 